\title{
Advances in the CAD-integrated Design, Analysis and Verification of Structural Membranes
}

\author{
A.-K. Goldbach* and K.-U. Bletzinger ${ }^{*}$ \\ * Technische Universität München \\ Lehrstuhl für Statik \\ Arcisstr. 21, 80333 München \\ e-mail: ann-kathrin.goldbach@tum.de, web page: https://www.bgu.tum.de/st
}

\begin{abstract}
The design and analysis of structural membranes requires the adjustment of the interdependent analyses of formfinding, structural analysis and cutting pattern generation - also called the design cycle. Furthermore, the verification of stability has to be performed on the basis of stress and deformation results computed in structural analysis. Due to the lightweight nature of structural membranes and their characteristic interaction of form and force, the analyses of the design cycle need to account for geometrical as well as material non-linearities.
\end{abstract}

CAD-integration enables designers and engineers to collaboratively work on one model for design and analysis purposes. The theory of Isogeometric Analysis was extended to Isogeometric B-Rep Analysis, in order to be able to assess the full B-Rep model within a CAD-environment for numerical analysis. This leads to the preservation of smooth NUBRS geometries and creates the possibility of conveniently linking consecutive analyses. Should changes of mechanical or geometrical model properties be necessary, these can automatically be forwarded to all design steps through these links. By additionally providing the possibility of working in a parametric framework and thus building models with parametric geometrical and mechanical properties, significantly enlarges the design space and exploration opportunities for structural membranes.

This paper highlights the advantages of the parametric CAD-integrated design cycle for structural membranes. The possibility of creating the necessary links between the individual design cycle steps facilitates design and analysis and furthermore allows for a fast and efficient incorporation of requirements arising from verification. Selected examples will show the vast design space and flexibility offered to architects and engineers by the presented methods. 